\title{
Velocity field and drag force measurements of a cube and a hemisphere mounted on an artificial bed surface roughness
}

\author{
Paride Nardone ${ }^{1, *}$ and Katinka Koll ${ }^{1}$ \\ ${ }^{1}$ Leichtweiß-Institute for Hydraulic Engineering and Water Resources, TU Braunschweig, \\ Braunschweig, Germany
}

\begin{abstract}
Quantification of the resistance in complex roughness situations, when both bed surface and form roughnesses contribute to the total resistance, as well as partitioning of the two contributions is still unsolved. Studies about form resistance of single elements focused on obstacles mounted on smooth bed surfaces, and only few considered a rough bed surface. In order to define an approach for shear stress partitioning in open channel flows, the effect of flow conditions, the geometrical characteristics of the obstacle, and the effect of the bed surface need to be studied. This paper contributes to the topic presenting results of experiments investigating the flow field around a cube and a hemisphere mounted on a bed surface with wake interference roughness. The velocity field and the drag force exerted on the obstacles were measured with a 3D Laser Doppler Anemometer and a drag force sensor, respectively. The double averaging methodology (DAM) was applied to define the characteristic region influenced by the cube and the hemisphere, and to analyse the streamwise velocities. DAM was developed for canopy flow, thus, the methodology needed to be adapted for isolated obstacle situations. A dependency of the drag coefficient on the relative submergence is observed and analysed.
\end{abstract}

\section{Introduction}

Flow resistance in fluvial streams has been the topic of many studies investigating either bed roughness or form roughness (e.g., [1]). In rivers with complex roughnesses, (e.g., gravel bed rivers, vegetated streams, flood plains) both the friction due to the roughness of the bed, as well as the drag due to the form of an obstacle, contribute substantially to resistance and need to be considered adequately. [2], for example, showed that bed friction contributed to the total shear stress by 5 to $25 \%$, depending on the density of artificial vegetation which varied between 11.1 and 44.4 plants $/ \mathrm{m}^{2}$. However, partitioning of the two contributions is still an unsolved question.

Most of the experiments focusing on form resistance were performed with unbounded objects (e.g., [3]). In case of wall-mounted objects, smooth bed conditions usually were

* Corresponding author: p.nardone@,tu-bs.de 
applied. [4] recently reviewed the literature describing the turbulent flow field around wallmounted objects, the effects of obstacle shape, and of relative submergence.

The influence of the shape of a body was investigated by, e.g., [5], showing that even small changes in the rounding of corners result in significant changes of the shedding characteristics, and thus of the form drag. However, they considered only smooth beds. [6] observed that the reattachment length downstream of an obstacle is shorter when mounted on a rough bed (made of small Lego bricks) than on a smooth bed. [7] described the flow characteristics induced by spheres placed on a rough bed made of glass spheres. They found that the form roughness of the obstacle is up to two times larger than the bed surface roughness in the boulder near wake region. The aforementioned studies were performed with rough beds, however, arranged in a densely packed way representing a quasi-smooth roughness.

This study investigates the drag force resulting from the combined effect of an isolated obstacle mounted on a wake interference bed surface roughness for different relative submergences. The velocity field with only the rough bed surface as well as with a single obstacle was measured with 3D laser Doppler anemometry (LDA). In order to consider the influence of the shape of the obstacle, a cube and a hemisphere were chosen. Drag forces measured with a drag force sensor (DFS) were used to calculate drag coefficients. Calculated and measured drag forces were compared to determine the domain for double averaging the velocities in case of the experiments with isolated obstacles. The streamwise velocities were used to analyse the characteristic approach velocity and its vertical position.

\section{Drag force and DANS}

The drag force $F_{D}$ exerted on an obstacle can be calculated with Eq. (1)

$$
F_{D}=C_{D} A_{f} \frac{\rho u_{c}^{2}}{2}
$$

where $C_{D}=$ drag coefficient, $A_{f}=$ frontal area of the obstacle, $\rho=$ fluid density, $u_{c}=$ characteristic approach velocity. $C_{D}$ depends on the separation characteristics of the obstacle boundary layer and is a function of the Reynolds number. In case of unbounded obstacles, $C_{D}$ decreases with increasing $R e$, and it is constant for $R e$ larger than 1000 , when the flow becomes turbulent. The relations for different geometries can be found in [3]. The given values are valid only for unbounded obstacles, or smooth bed conditions (e.g., [9]), where $u_{c}$ is usually assumed to be equal to the bulk velocity. This velocity is no longer appropriate in case of rough bed surfaces (e.g., [7]), and especially in flow over and through complex roughnesses (e.g., [11]).

In order to investigate flows over rough beds the double averaged Navier-Stokes equations are a suitable methodology ([12]). Spatial averaging of time-averaged hydrodynamic variables can adequately describe the spatial flow heterogeneity. For twodimensional, steady, uniform flow conditions over a fixed rough bed, the longitudinal component of the double averaged momentum equation can be written as ([13]),

$$
\rho g S_{b}+\frac{1}{\phi} \frac{\partial \phi \tau}{\partial z}-f_{d}=0
$$

where, $g=$ gravity acceleration, $S_{b}=$ bed slope, $\phi=$ roughness geometry function, $z=$ vertical coordinate. $f_{d}$ is the drag force per unit fluid volume. It can also be expressed in terms of shear stress when considered acting on a surface, and compared with the fluid shear stress $\tau$, which contains Reynolds, form induced, and viscous stresses:

$$
\tau=\rho\left[-\left\langle\overline{u^{\prime} w^{\prime}}\right\rangle-\langle\tilde{u} \tilde{w}\rangle+\frac{v}{\phi} \frac{d \phi\langle\bar{u}\rangle}{d z}\right]
$$


where $\langle\bar{u}\rangle=$ double averaged longitudinal velocity component, $u^{\prime}$ and $w^{\prime}=$ longitudinal and vertical temporal fluctuation, $\tilde{u}$ and $\tilde{w}=$ longitudinal and vertical spatial fluctuation, and $v=$ kinematic viscosity. The bed shear stress $\tau_{0}$ is obtained by integrating Eq. (2) (see [12])

$$
\begin{aligned}
& \frac{\tau_{0}(x, \Lambda)}{\rho}=\int_{z_{t}}^{z_{c}} f_{d} d z=g S_{b}\left[z_{w s}-z_{c}+\int_{z_{t}}^{z_{c}} \phi d z\right]- \\
& -\int_{z_{t}}^{z_{w s}} \frac{\partial \phi\left\langle\overline{u^{\prime} w^{\prime}}\right\rangle}{\partial z} d z-\int_{z_{t}}^{z_{w s}} \frac{\partial \phi\langle\tilde{u} \tilde{w}\rangle}{\partial z} d z+\int_{z_{t}}^{z_{w s}} \frac{\partial}{\partial z} \phi\left\langle v \frac{\partial \bar{u}}{\partial z}\right\rangle d z
\end{aligned}
$$

where $x$ =streamwise coordinate, $z_{c}=$ roughness crest, $z_{t}=$ minimum bed elevation, $z_{w s}=$ water surface, and $\Lambda=$ horizontal (plane) size of the averaging window. The explicit dependence of $\tau_{0}$ on the averaging domain, suggests that Eq. (4) can be applied as a base for the shear stress partitioning ([12]). However, first, the averaging domain needs to be defined, being large enough to fulfil the request of the DANS methodology, i.e., the 2D uniform flow conditions, and smaller than the scales of the mean flow.

\section{Experiments}

\subsection{Experimental setup}

The experiments were carried out in a $32 \mathrm{~m}$ long, $0.6 \mathrm{~m}$ wide, and $0.4 \mathrm{~m}$ deep tilting flume. The bed surface was made of a plastic sheet, with smooth edged cone shaped roughness elements (Fig. 1a). The cones had a geometrical height $k=8 \mathrm{~mm}, 8 \mathrm{~mm}$ spaced, and with a diameter between $16 \mathrm{~mm}$ and $8 \mathrm{~mm}$ at the base and at the top of each element, respectively, resulting in a wake interference roughness, which is characterised and described in detail in [8]. The rough bed surface covered a length of $10 \mathrm{~m}$ upstream of the $1.5 \mathrm{~m}$ long measuring section, located at the middle of the flume, and further $5 \mathrm{~m}$ downstream of it.

A cube with $30 \mathrm{~mm}$ lateral length and a frontal area $A_{f}=9 \mathrm{~cm}^{2}$, and a hemisphere with a diameter of $60 \mathrm{~mm}$ and $A_{f}=14 \mathrm{~cm}^{2}$ (Fig. 1b, c), were used to represent geometrically different obstacles with smooth and sharp edges, but with the same geometrical height $k_{e}$.

The velocity was measured with a Dantec 3D Laser Doppler Anemometer with a 2W Argon-Ion laser. The two watertight $27 \mathrm{~mm}$ probes, with a focal length of $198 \mathrm{~mm}$ in water, were installed on an automatic traverse system, located above the measuring section. A false bottom allowed to install the DFS below the bed (Fig. 1d).

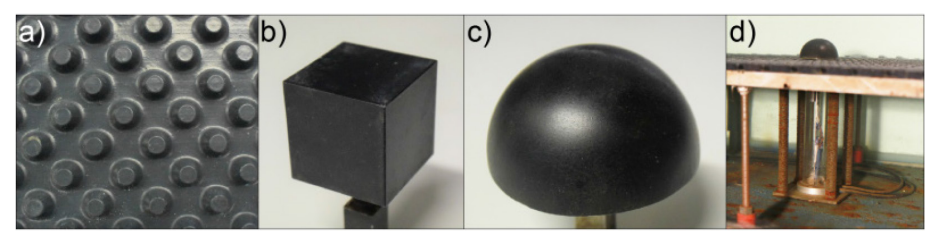

Fig. 1. a) Cones bed surface roughness; b) cube; c) hemisphere; c) installation of the DFS underneath the false bottom of the flume.

\subsection{Experimental procedure}

Drag force measurements were performed with five relative submergences $h / k_{e}$. A fixed bed slope $S_{b}=0.0005$ was considered, and the discharge $Q$ was adapted in order to have 
steady uniform flow conditions. The water surface level was measured with point gauges and the slope was calculated from linear regression. The experimental parameters are summarized in Table 1.

Table 1. Hydraulic flow conditions.

\begin{tabular}{|c|c|c|c|c|c|}
\hline$h[\mathrm{~m}]$ & $h / k_{e}[-]$ & $Q\left[\mathrm{~m}^{3} / \mathrm{s}\right]$ & $u_{m}[\mathrm{~m} / \mathrm{s}]$ & $\operatorname{Re}[-]$ & $u *[\mathrm{~m} / \mathrm{s}]$ \\
\hline 0.15 & 5.0 & 0.031 & 0.34 & $1.03 \mathrm{E}+04$ & 0.027 \\
\hline 0.20 & 6.7 & 0.045 & 0.37 & $1.11 \mathrm{E}+04$ & 0.031 \\
\hline 0.25 & 8.3 & 0.065 & 0.43 & $1.30 \mathrm{E}+04$ & 0.035 \\
\hline 0.30 & 10.0 & 0.093 & 0.51 & $1.54 \mathrm{E}+04$ & 0.038 \\
\hline
\end{tabular}

with $u_{m}=$ bulk velocity, $R e_{e}=u_{m} k_{e} / v=$ Reynolds number, $u *=\left(g h S_{b}\right)^{-0.5}=$ friction velocity.

The drag force $F_{D}$ exerted on the two obstacles was measured with a sampling period of $60 \mathrm{~s}$ and a sampling frequency of $1614 \mathrm{~Hz}$. Details about the drag force sensor can be found in [14].

Measurements of the detailed flow field were performed with $h / k_{e}=5$ and 10 . The origin of the vertical coordinate $(z=0)$ was set at the minimum bed elevation. Velocity measurements started at $z=1.1 \mathrm{~cm}, 0.3 \mathrm{~cm}$ above the bed surface roughness crests, in order to avoid interferences due to light reflection. Point measurements were carried out with a vertical spacing $\Delta z=0.2 \mathrm{~cm}$ up to $z=4 \mathrm{~cm}, \Delta z=0.5 \mathrm{~cm}$ up to $20 \%$ of the water depth, and $\Delta \mathrm{z}=1.0 \mathrm{~cm}$ up to $z=11 \mathrm{~cm}$. The sampling duration at each point was $2 \mathrm{~min}$, and the sampling frequencies ranged between 20 and $120 \mathrm{~Hz}$.

For the rough bed without obstacle, 21 vertical velocity profiles were measured in planes parallel to the bed. The profiles were equally spaced by $1.7 \mathrm{~cm}$ in $x$ and $y$ direction and with a minimum distance of $28 \mathrm{~cm}$ to the walls. The measuring grid used in case of cube and hemisphere, mounted on the rough bed surface, is represented in Fig. 2.

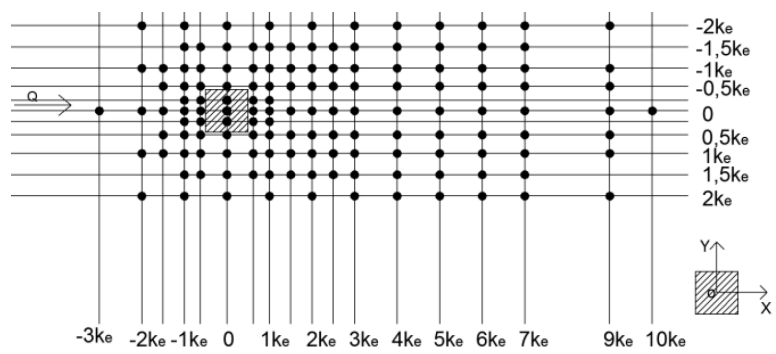

Fig. 2. Velocity measuring grid adopted for combined bed surface and form roughnesses.

The origin of the reference system is located at the middle of the obstacle, and the location of the points in $x$ and $y$ direction is related to the geometrical height $k_{e}$.

\section{Results}

\subsection{Drag force and drag coefficient}

The drag forces measured for cube and hemisphere, at different relative submergences, are represented in Fig. 3a. Despite the different size of the frontal area, which is larger in case of the hemisphere, the two obstacles present similar $F_{D}$ values. This is related to their geometry, where the sharp edges of the cube increase the flow separation, resulting in a 
larger $F_{D}$ (e.g., [5]). Values of $C_{D}$, presented in Fig. 3b, were calculated with Eq. (1) using the measured $F_{D}$ and the bulk velocity $u_{m}$ as characteristic velocity. The $C_{D}$ values in case of the cube are larger than the ones of the hemisphere. This is related to the geometry of the obstacles and it is confirmed by studies reported in literature (e.g., [3]). However, despite the turbulent flow conditions, $C_{D}$ decreases with increasing $h / k_{e}$. In fact, within the considered range of Reynolds numbers (Tab. 1), the drag coefficient should be constant, with $C_{D}=1.05$ for the cube and 0.40 for the hemisphere (e.g., [3]). This difference can be attributed to the presence of the bed surface roughness and $u_{m}$, which is not always the most suitable velocity to be used in Eq. (1).
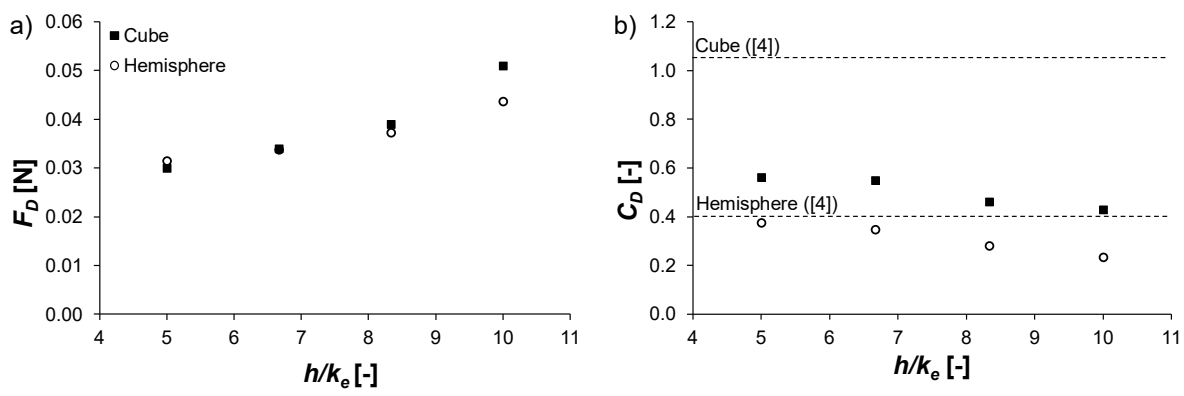

Fig. 3. a) Drag force measurements and b) drag coefficients for cube and hemisphere, mounted on a rough bed surface, at different levels of relative submergence.

\subsection{Analysis of the flow field and application of DANS}

In order to analyse the velocity data, the grid was refined to a resolution of $0.125 k_{e}$ by linear interpolation, obtaining a uniform grid with equidistant points.

The definition of the averaging domain for the flow field with obstacle is based on the relative difference $\Delta u$ between the streamwise velocity component in conditions with and without obstacle (Eq. 5). The flow field over the homogeneously rough surface without obstacle is characterised by the double averaged vertical profile of the velocity calculated by averaging the 21 measured profiles in planes parallel to the bed, obtaining $\left\langle\bar{u}_{n o_{-} \text {elem }}\right\rangle$.

$$
\Delta u=\frac{\left|\bar{u}_{\text {elem }}-\left\langle\bar{u}_{\text {no_elem }}\right\rangle\right|}{\left\langle\bar{u}_{\text {no_elem }}\right\rangle}
$$

where $\bar{u}_{\text {elem }}=$ time averaged velocity for the case with the presence of the obstacle. Thus, a map of relative differences is obtained for each averaging plane. The analysed area was located $0.3 \mathrm{~cm}$ above the bed surface roughness crest, at $z=1.1 \mathrm{~cm}$, where both surface and form roughnesses influence the flow field.

Fig. 4 shows contour plots of the velocity differences influenced by the cube and the hemisphere, respectively. The region affected by the cube was larger than the one of the hemisphere, because of the flow separation generated by the sharp edges of the cube. The generated turbulent structures were stronger than the ones in case of the hemisphere, and they develop for a longer distance.

The region influenced by the hemisphere extends up to $x / k_{e}=6$, being shorter than the one reported in, e.g., [15], where $x / k_{e}=10$ was determined in experiments with quasismooth bed roughnesses. The wake interference roughness of the bed surface used in the present study reduced the region influenced by the form roughness. This effect was already observed in wind tunnel experiments of [6] comparing a smooth and a rough bed. 
The larger relative submergence resulted in smaller areas affected by the obstacles in case of the cube and the hemisphere. According to, e.g., [4], the base vortices generated by the form roughness are attenuated by the larger relative submergence.

a)

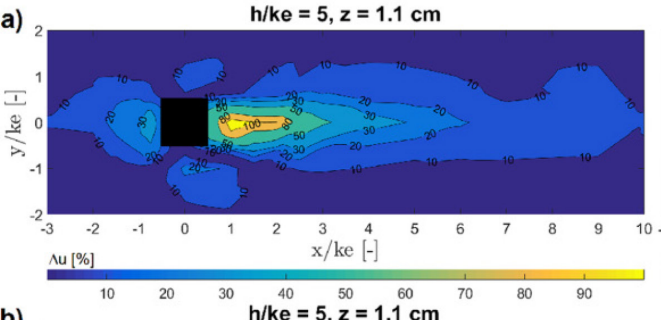

b)

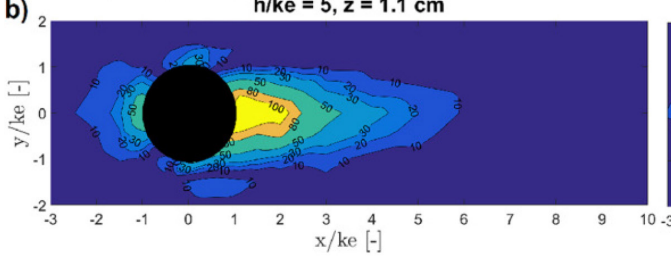

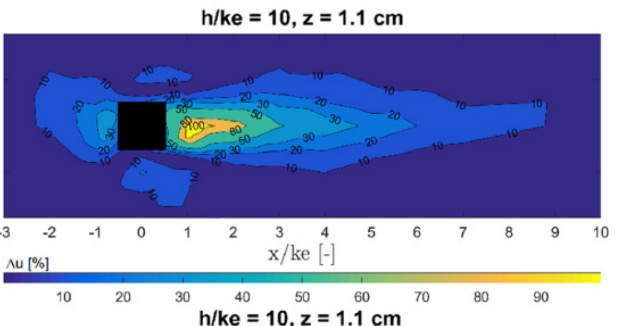

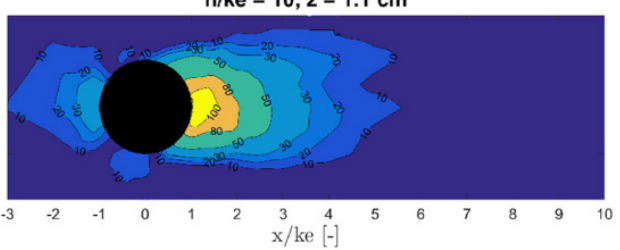

Fig. 4. Contour plot of the area influenced by a) the cube and b) the hemisphere.

In order to define the characteristic region influenced by the form roughness, the DAM was applied in different averaging domains determined by $\Delta u$. After choosing a $\Delta u$ in the plane closest to the bed roughness crest, i.e. at $z=1.1 \mathrm{~cm}$, the limits of the contour line in $\mathrm{x}$ and $y$ direction were determined, giving the length and width of the rectangular averaging area. The rectangle had to be symmetric along the spanwise coordinate to fulfil the $2 \mathrm{D}$ flow conditions within the averaging domain. Using the determined area for spatial averaging, first, the shear stress was calculated with Eq. (4) and afterwards $F_{D}$ was calculated with

$$
F_{D}=\Lambda \int_{z=k}^{z=k_{e}} \rho \phi f_{d} d z
$$

Finally, the calculated drag forces were compared to the measured $F_{D}$ values. The resulting values of $\Delta u$ and $\Lambda$ as well as the calculated and measured values of $F_{D}$ are presented in Table 2 together with further relevant parameters. Remaining differences between measured and calculated $F_{D}$ may be attributed to the stepwise increase of $\Delta u$.

Table 2. Geometry of characteristic averaging domains, and calculated $F_{D}$.

\begin{tabular}{|c|c|c|c|c|c|c|c|c|c|}
\hline $\begin{array}{c}\text { Form } \\
\text { roughness }\end{array}$ & $\begin{array}{c}A_{f} \\
{\left[\mathrm{~m}^{2}\right]}\end{array}$ & $\begin{array}{c}h / k_{e} \\
{[-]}\end{array}$ & $\begin{array}{l}\Delta u \\
{[\%]}\end{array}$ & $\begin{array}{c}x / k_{e} \text { upstream } \\
{[-]}\end{array}$ & $\begin{array}{c}x / k_{e} \text { downstream } \\
{[-]}\end{array}$ & $\begin{array}{c}y / k_{e} \\
{[-]}\end{array}$ & $\begin{array}{c}\Lambda / A_{f} \\
{[-]}\end{array}$ & $\begin{array}{c}F_{D \text { meas. }} . \\
{[\mathrm{N}]}\end{array}$ & $\begin{array}{c}F_{D \text { calc }} \\
{[\mathrm{N}]}\end{array}$ \\
\hline \multirow[t]{2}{*}{ Cube } & \multirow[t]{2}{*}{0.0009} & 5 & 5 & -3.0 & 10.0 & 1.9 & 50 & 0.031 & 0.035 \\
\hline & & 10 & 10 & -2.3 & 8.8 & 1.5 & 33 & 0.051 & 0.050 \\
\hline \multirow[t]{2}{*}{ Hemisphere } & \multirow[t]{2}{*}{0.0014} & 5 & 5 & -3.0 & 8.0 & 2.0 & 28 & 0.032 & 0.029 \\
\hline & & 10 & 5 & -3.0 & 6.0 & 2.0 & 23 & 0.044 & 0.044 \\
\hline
\end{tabular}

The sizes of the averaging domain necessary for matching measured and calculated $F_{D}$ were quite large as the streamwise velocities in the experiments with obstacles were allowed to deviate only by $5 \%$ (in one case $10 \%$ ) from the undisturbed velocities.

As a consequence of the form roughness shape, the normalised averaging domain was larger for the cube than for the hemisphere, independent of $h / k_{e}$. The upstream extension of the wake zone was almost unaffected by the shape of the obstacle and the relative submergence. However, due to the larger relative submergence the length of the downstream far-wake zones was reduced. As the size of the wake zone is related to the drag 
coefficient, it can be expected that the $C_{D}$ value depends on $h / k_{e}$, which is presented in Fig. 3. However, together with $h / k_{e}$ also the characteristic approach velocity $u_{c}$ changes.

Usually the bulk velocity is used together with a constant $C_{D}$ value to calculate the drag force (see Eq. 1). Another suggestion is to use the velocity in front of the obstacle and in $50 \%$ of its height (e.g., $[7,10])$.

Comparing measured and calculated $F_{D}$ values, the required approach velocities $u_{c}$ and their vertical position can be determined keeping $C_{D}$ constant and according to literature ([3]). $u_{c}$ was obtained considering three methods: (1) double averaged velocity in case of bed roughness without obstacle; (2) double averaged velocity with cube and hemisphere, respectively (averaging domains see Table 2); (3) the velocity profile measured in front of the obstacle, at $x / k e=-0.5$ for the cube, and $x / k e=-1.0$ for the hemisphere.

The velocities $u_{c}$ requested to match measured and calculated $F_{D}$ were smaller than the bulk velocities $u_{m}$. In fact, the bulk velocity is characteristic only for unbounded obstacles, as it is not affected by walls (e.g., [3]). Close to the walls the velocity is decreased and thus, even for obstacles mounted on smooth beds $u_{m}$ is no longer characteristic. With increasing roughness of the bed surface the velocity relevant for the drag force is even smaller.

The vertical positions of the requested velocities considering the aforementioned methods are presented in Fig. 5.
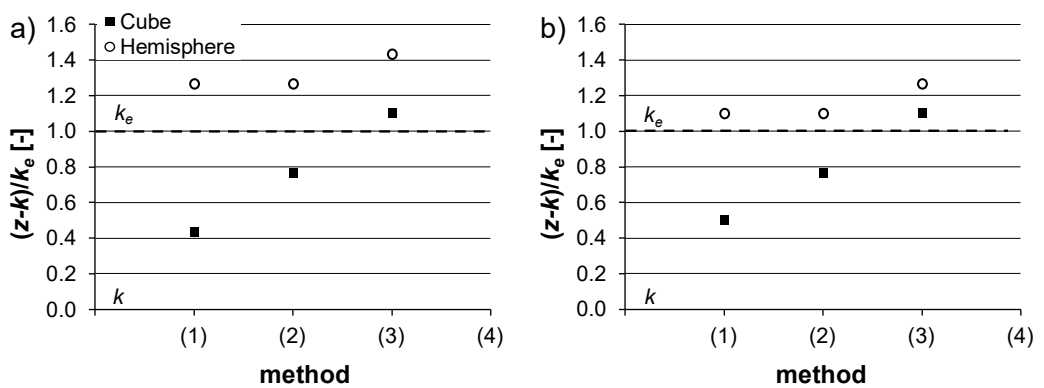

Fig. 5. Vertical position of approach velocity $u_{c}$ depending on the method for determination of the vertical velocity profile, a) $h / k_{e}=5$, b) $h / k_{e}=10$.

For the hemisphere the characteristic velocity was always positioned above the crest of the obstacle. The height was independent of the method when using the undisturbed or disturbed double averaged velocity profile, however, using the local velocity profile in front of the obstacle the vertical position of the characteristic approach velocity is somewhat higher. Due to the increased relative submergence the vertical position is shifted towards the crest of the obstacle. The recommendation of [10], measuring $u_{c}$ in front of a hemisphere in $50 \%$ of its height, cannot be confirmed. This may be an effect of the bed surface roughness which was quasi-smooth in the experiments of [10]. The larger roughness of the wake interference bed surface, used here, resulted in lower velocities close to the bed, which is not fully reflected in the resulting drag force, and thus the position of $u_{c}$ had to be further away from the bed surface.

For the cube the vertical position of $u_{c}$ was independent of $h / k_{e}$ but depended on the method. Using the double averaged profile of the undisturbed flow field (method 1) the characteristic velocity was at about $50 \%$ of the height of the obstacle, the double averaged profile of the disturbed flow field (method 2) resulted in a position of $77 \%$ of $k_{e}$, and method 3 (velocity profile just in front of the obstacle) required a position above the obstacle. One reason for the different results for the hemisphere and the cube can be found in the difference between undisturbed and disturbed double averaged profiles which was higher for the cube. Further analyses are in progress. 


\section{Summary and conclusions}

The drag force $F_{D}$ of a cube and a hemisphere, respectively, mounted on a bed surface with wake interference roughness was measured as well as calculated using the double averaged streamwise velocity for two relative submergences $h / k_{e}$.

According to literature, $F_{D}$ was higher for the cube than for the hemisphere. However, calculating the drag coefficient with measured $F_{D}$ showed a dependency of $C_{D}$ on $h / k_{e}$. In order to analyse the characteristic approach velocity $u_{c}$, the double averaged streamwise velocity was used. A method to determine the double averaging domain for an isolated obstacle is presented.

By comparing measured and calculated $F_{D}$ values, keeping $C_{D}$ constant and according to literature, $u_{c}$ and its position above the bed surface were determined. The recommendation of using the velocity in front of an obstacle and in $50 \%$ of its height could not be confirmed. This can partly be attributed to the lower velocities close to the bed due to the wake interference roughness, used here, compared to the quasi-smooth bed surface used to develop the recommendation. Moreover, the vertical position of $u_{c}$ was not constant but depended on $h / k_{e}$. Further analyses of the flow field are in progress integrating data from experiments with varying bed surface roughness to investigate the relation between the drag force, the bed roughness, and characteristic parameters.

Acknowledgments: This work was supported by the 7th Framework Programme of the European Union, (Marie Curie - FP7-PEOPLE-2012-ITN), which funded the Initial Training Network (ITN) HYTECH 'Hydrodynamic Transport in Ecologically Critical Heterogeneous Interfaces', N. 316546.

\section{References}

1. D. M. Powell, Earth-Science Rev., 136, 301-338 (2014)

2. T. Schoneboom, J. Aberle, A. Dittrich, Proc. Int. Conf. Fluv. Hydr., 269-276 (2010)

3. F. M. White, Fluid Mechanics (2011)

4. S. M. Hajimirzaie, C. J. Wojcik, J. H. J. Buchholz, Exp. Fluids, 53, 1943-1962 (2012)

5. S. Okamoto, N. Uemura, Exp. Fluids, 11, 58-64 (1991)

6. E. Savory, N. Toy, J. Wind Eng. Ind. Aerodyn., 23, 345-364 (1986)

7. N. Papanicolaou, C. M. Kramer, A. G. Tsakiris, T. Stoesser, S. Bomminayuni, Z. Chen, Acta Geophysica, 60, 6, 1502-1546 (2012)

8. P. Nardone, Ka. Koll, Springer series: GeoPlanet: Earth Planetary Scienc. $34^{\text {th }}$ ISH, 235-244 (2006)

9. M. Oertel, S. Peterseim, A. Schlenkhoff, J. Hydraul. Res., 49, 3, 372-377 (2011)

10. N. L. Coleman, Proc. 12th IAHR Congress, 185-192 (1967)

11. A. Armanini, M. Rughetti, P. Grisenti, J. Hydraul. Res., 43, 5, 481-487 (2005)

12. V. Nikora, I. McEwan, S. McLean, S. Coleman, D. Pokrajac, R. Walters, J. Hydraul. Eng., 133, 8, 873-883 (2007)

13. V. Nikora, S. McLean, S. Coleman, D. Pokrajac, I. McEwan, L. Campbell, J. Aberle, D. Clunie, Ka. Koll, J. Hydraul. Eng., 133, 8, 884-895 (2007)

14. T. Schoneboom, J. Aberle, C. A. M. E. Wilson, A. Dittrich, Proc. 8th Int. Conf. Hydr.Scienc. Eng. (2008)

15. H. Shamloo, N. Rajaratnam, C. Katopodis, J. Hydraul. Res., 39, 4, 351-366 (2001) 